\title{
Diagnóstico de masa inguinal, más allá de la hernia inguinal
}

\author{
Diagnosis of inguinal mass, more than inguinal hernia
}

\author{
María Trigás Ferrín, Lucía Ferreira González \\ Servicio de Medicina Interna. Hospital Arquitecto Marcide. SERGAS. Ferrol
}

\section{Resumen}

Caso clínico de mujer de 86 años con masa inguinal izquierda como hallazgo incidental en la exploración física y diagnóstico final de quiste de canal de Nuck, una rara entidad en adultos y que obliga a revisar el diagnóstico diferencial de las masas inguinales.

Palabras clave: Quiste de canal de Nuck. Masa inguinal. Diagnóstico diferencial

\section{Introducción}

El canal de Nuck está formado por una pequeña evaginación del peritoneo parietal a través del anillo inguinal hacia el interior del canal inguinal, acompañando al ligamento redondo en las mujeres y que equivale al proceso vaginalis en los hombres. Su ausencia de cierre, que habitualmente tiene lugar durante el primer año de vida, da lugar a la aparición de hernias inguinales indirectas o hidroceles ${ }^{1,2,3}$. Los hidroceles o quistes del canal de Nuck están formados por el acúmulo de líquido peritoneal a ese nivel. El diagnóstico de estas patologías se realiza principalmente en la infancia siendo un diagnóstico menos frecuente en la edad adulta y excepcional en la senectud.

\section{Caso clínico}

Presentamos el caso de una mujer de 86 años con antecedentes personales de hipertensión arterial, dislipemia, insuficiencia renal crónica estadio 3, insuficiencia mitral moderada, fibrilación auricular paroxística y colecistectomía. Tres años antes se había realizado estudio con TAC por cuadro de dolor abdominal donde presentaba quistes hepáticos simples y dilatación de la vía biliar intrahepática y del colédoco secundaria a cirugía previa de vesícula.

Acude al hospital por cuadro de dolor abdominal localizado a nivel de hipogastrio, estreñimiento y meteorismo de años de evolución que se ha intensificado en las últimas horas. Sin presencia de náuseas, vómitos ni clínica miccional y con ausencia de tos, disnea u otra sintomatología por aparatos.

En la exploración física destaca una temperatura de $38^{\circ} \mathrm{C}$, saturación de oxígeno de $88 \%$ y crepitantes a nivel de base pulmonar derecha. En la exploración abdominal presenta abdomen depresible, con molestia a la presión profunda sobre hipogastrio, sin presencia de masas ni organomegalias y con ruidos abdominales normales. En la región inguinal izquierda se palpa una masa bien delimitada, redondeada, no dolorosa, de pequeño tamaño y consistencia elástica que no se modifica con la tos ni con los cambios posturales.

\begin{abstract}
A report of a 86-year-old female with an incidental groin mass found to be a cyst of the canal of Nuck is presented. This rare finding serves to make the differential diagnosis for common groin masses.

Keywords: Cyst of the canal of Nuck. Groin mass. Differential diagnoses
\end{abstract}

En la analítica se aprecia leucocitosis con desviación izquierda, insuficiencia renal ya conocida y valores de transaminasas y amilasa normales. En la radiografía de tórax presenta un infiltrado pulmonar localizado a nivel del lóbulo medio y en la radiografía de abdomen luminograma normal con ateromatosis aórtica calcificada. Se realiza TAC abdominal con presencia de dilatación de vía biliar intra/ extrahepática, ya presente en estudio previo, ausencia de líquido libre peritoneal ni datos de diverticulitis. Se describe una adenopatía a nivel inguinal izquierdo de $1.7 \mathrm{~cm}$. de diámetro.

Ingresa en el hospital con el diagnóstico de neumonía comunitaria y se inicia tratamiento antibiótico presentando evolución clínica y radiológica favorable. Durante su estancia en el hospital la paciente no refiere síntomas digestivos excepto estreñimiento habitual. Se solicita determinación de marcadores tumorales con CEA, CA 19.9, CA 125 y alfafetoproteína normal y ecografía de zona inguinal izquierda para valoración de la lesión descrita en la exploración física. El estudio ecográfico identifica una lesión anecoica de $2.3 \mathrm{~cm}$. de diámetro máximo con un fino cuello en su vertiente posterior que

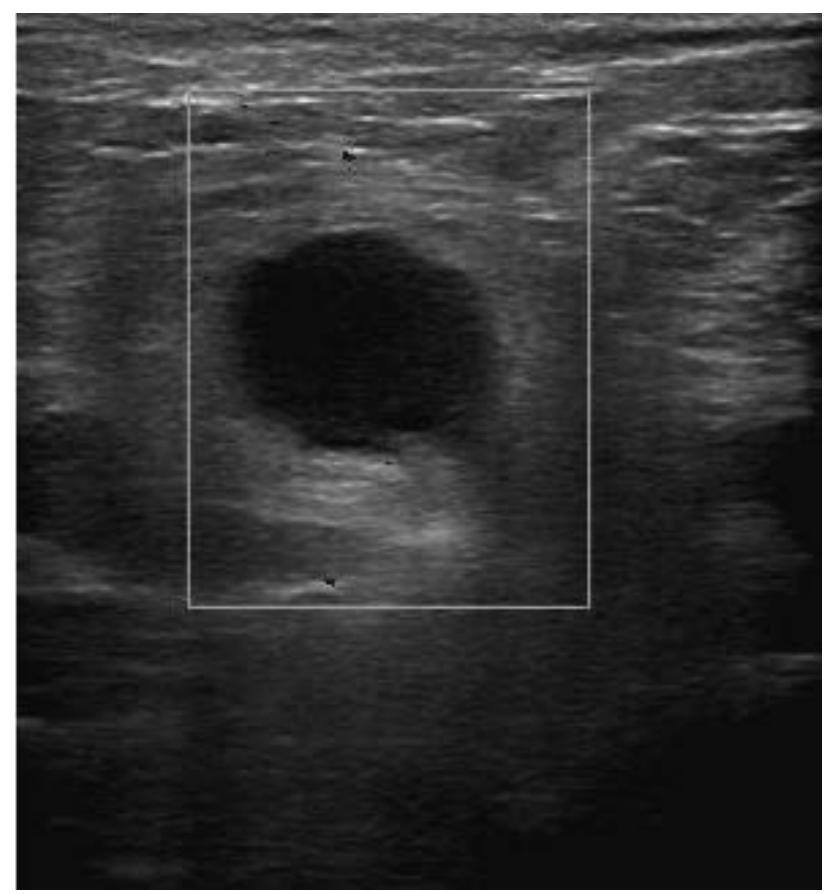


Tabla 1. Diagnóstico diferencial entre hidrocele de canal de Nuck y hernia inguinal indirecta.

\begin{tabular}{|l|c|c|}
\hline & Hidrocele canal de Nuck & Hernia inguinal indirecta \\
\hline Dolor & Indolora o leve & Indolora o dolor si existe complicación \\
Síntomas abdominales & No & Náuseas, vómitos \\
Ruidos abdominales & No & Sí \\
Posición supina & No desaparece & Desaparece (aparece en bipedestación) \\
Maniobra de Valsalva & No se modifica & Se modifica \\
Transiluminación & Positiva (en lesiones grandes) & Negativa (presencia de contenido abdominal) \\
\hline
\end{tabular}

parece continuarse hacia el peritoneo sin mostrar contenido en su interior y que no se modifica con las maniobras de Valsalva ni presenta flujo vascular con el estudio doppler color siendo sugestivo de quiste del canal de Nuck (Imagen 1). Se revisan las imágenes de las dos tomografías abdominales realizadas previamente y en ambas se identifica dicha lesión en solución de continuidad con el peritoneo y sin cambios en el tamaño ni en la morfología.

Teniendo en cuenta la edad avanzada de la paciente, la comorbilidad asociada y la ausencia de correlación entre la clínica y los hallazgos radiológicos, se decidió actitud conservadora y no se valoró tratamiento quirúrgico de la lesión.

\section{Discusión}

En 1691 el anatomista holandés Anton Nuck describió por primera vez una pequeña evaginación del peritoneo vaginal que acompañaba al ligamento redondo de las mujeres a través del anillo inguinal al que se denominó posteriormente canal de Nuck4 . Dicha evaginación desaparece habitualmente durante los primeros 8 meses de gestación y su persistencia puede dar lugar a la aparición de hernias inguinales (si existe el paso de vísceras al espacio extrabdominal) o quistes del canal de Nuck (si existe paso de fluido peritoneal). Los quistes de canal de Nuck son infrecuentes, la mayoría de los casos se han descrito en neonatos o niños, siendo una rara condición en adultos ${ }^{5}$.
El diagnóstico de hidrocele o quiste de canal de Nuck rara vez es posible realizarlo únicamente con los hallazgos clínicos. Los pacientes habitualmente refieren la existencia de una masa indolora o ligeramente dolorosa localizada a nivel de región inguinal, fluctuante, no reducible y sin síntomas abdominales agudos asociados ${ }^{6}$. Suelen ser de pequeño tamaño, raramente sobrepasan los cinco centímetros y pueden desplazarse hacia los labios mayores, siendo posible en estos casos la realización de transiluminación que ayudaría al diagnóstico ${ }^{7}$. En pacientes obesos puede ser imposible definir la localización del quiste a nivel del anillo inguinal por pérdida de las referencias anatómicas. Se acepta que el anillo inguinal interno se localiza aproximadamente en la zona media entre la espina ilíaca anterosuperior y la sínfisis púbica ${ }^{8}$. El diagnóstico también puede ser difícil en aquellos casos en los que coexista una hernia inguinal (30-40\%)

El diagnóstico diferencial de masas inguinales en mujeres es amplio e incluye hernias inguinales y femorales, adenopatías, tumores de partes blandas (lipomas, leiomiomas y endometriosis), quistes de Bartholino y menos frecuentes lesiones vasculares y abscesos ${ }^{6}$.

El diagnóstico diferencial se debe realizar principalmente con las hernias inguinales teniendo en cuenta que ambas

Tabla 2. Diagnóstico diferencial ecográfico de masas inguinales

\begin{tabular}{|l|l|}
\hline Hidrocele Canal de Nuck & $\begin{array}{l}\text { Anecoica de tamaño variable }(<5 \mathrm{~cm} .) \\
\text { No reducible } \\
\text { No se modifica con maniobras de Valsalva } \\
\text { Ausencia de contenido abdominal } \\
\text { Señal doppler negativa }\end{array}$ \\
\hline Hernia inguinal & $\begin{array}{l}\text { Hiperecoica de tamaño variable ( puede ser }>5 \mathrm{~cm} .) \\
\text { Reducible } \\
\text { Se modifica con maniobras de Valsalva } \\
\text { Presencia de contenido abdominal } \\
\text { Señal doppler negativa }\end{array}$ \\
\hline Adenopatías & $\begin{array}{l}\text { Hiperecoicas aunque linfomas malignos pueden tener al principio aspecto anecoico } \\
\text { Señal doppler muy positiva por abundante vasculatura }\end{array}$ \\
\hline Abscesos & Pared irregular hipoecoica con contenido ecogénico tipo detritus o gas \\
\hline Lesiones vasculares & Señal doppler positiva \\
\hline
\end{tabular}


entidades pueden coexistir en un tercio de los pacientes. Desde el punto de vista clínico la ausencia de dolor y síntomas abdominales asociados, la ausencia de modificación del tamaño de la masa con la bipedestación o con las maniobras de Valsalva y una transiluminación positiva, apoyan el diagnóstico de hidrocele. Por otro lado, la presencia de ruidos abdominales sobre la lesión orienta hacia el diagnóstico de hernia inguinal ${ }^{9}$. En la tabla 1 se resumen los hallazgos clínicos de ambas entidades.

La ecografía es la técnica diagnóstica de elección en el estudio de masa inguinal en las mujeres y se recomienda su realización de forma rutinaria dado que es una técnica sencilla, accesible y de alto rendimiento diagnóstico ${ }^{9}$. En la ecografía los quistes del canal de Nuck se presentan como una lesión quística localizada en la región inguinal, bien delimitada, con morfología variable en función de la disposición que adopte dentro del canal inguinal (alargada, en forma de coma, quiste dentro de quites, redondeada o en reloj de arena), que no se modifica con las maniobras de Valsalva y con ausencia de contenido abdominal y señal doppler en su interior $^{6,7}$. En la tabla 2 se resume el diagnóstico diferencial ecográfico de estas lesiones.

La RMN puede aportar imágenes más precisas, informar sobre la presencia de septos o comunicación con el peritoneo, y dar información sobre las relaciones anatómicas con las estructuras adyacentes. En resonancia magnética se presenta como una lesión de paredes finas, con baja intensidad en T1 y señal alta en T2 por su contenido líquido que no realza con la administración de gadolinio ${ }^{7,9}$.

En la TAC se visualiza como una masa redondeada en la región inguinal con atenuación homogénea que no realza con contraste. Las imágenes axiales son poco específicas y a menudo se confunden con adenopatías tumorales o infla- matorias. Las reconstrucciones multiplanares, especialmente sagitales, ponen de manifiesto un fino pedículo que se dirige hacia la cavidad peritoneal a través del anillo inguinal? . El diagnóstico definitivo se realiza durante la cirugía y se confirma con el examen anatomopatológico donde se pone de manifiesto la existencia de tejido peritoneal. En algunos casos se han descrito modificaciones del epitelio con presencia de endometriosis ${ }^{10}$, angiomiofriboma ${ }^{11}$ e incluso un caso de adenocarcinoma ${ }^{12,13}$.

El tratamiento de elección es el quirúrgico con resección del hidrocele y ligadura del cuello del proceso vaginalis ${ }^{9}$.

\section{Bibliografía}

1. Anderson CC, Broadie TA, Mackey JE, Kopecky KK. Hidrocele of the canal of Nuck: ultrasound appearance. Am Surg 1995, 61: 959-961

2. Schwartz A, Peyser MR. Nuck's hydrocele ( hydrocele muliebris). Int Surg 1975; 60: $91-92$

3. Counseller VS, Marden B. Hydrocele of the canal of Nuck. Annals of Surgery 1941, 113 (4): 625-630

4. Nuck A: Adenographia curiosa et uteri foeminei anatome nova. Leiden, Luchtmans, 1691, p 130)

5. Caviezel A, Montet X, Schwartz J, Egger J-F, Iselin CE. Female hidrocele: the Cyst of Nuck. Urol Int 2009,82: 242-245

6. Stickel WH, Manner M. Female Hydrocele ( Cyst of the Canal of Nuck). Songraphic Appearance of a Rare and Little-Known Disorder. J Ultrasound Med 2004; 23: 429 432

7. Beltrán M, Mayayo E, Angulo E. Solution to case 18: Hidrocele of the canal of Nuck. Radiología. 2010; 52(3):270-272

8. Hale S, Mirjalili S, Stringer M. Inconsistencies in Surface Anatomy: The Need of an Evidence-Based Reapprasial. Clinical Antomy 2010; 23: 922-930

9. Yu Mi Choi, Gyu Min Lee, Jung Bin Yi et al. Two cases of female hydrocele of the canal of nuck. Korean J Pediatr 2002; 55 (4): 143-146

10. Cervini P, Mahoney J, Wu L: Edometriosis in the canal of Nuca: atypical manifestations in an inusual location. AJR Am J Roentgenol 2005; 185: 284-285

11. Laskin WB, Fetsch JF, Tavassoli FA: Angiomyofibroblastoma of the female genital tract: análisis of 17 cases including a lipomatous variant. Hum Pathol 1997,28: 1046-1055

12. MeskoJD, Gates $\mathrm{H}$, et al: Clear cell adenocarcinoma of the vulva arising in endometriosis : a case report. Gynecol Oncol 1988; 29: 385-391

13. Sun CC, Toker C, Masi JD, et al: Primary low grade adenocarcinoma occurring in the inguinal region. Cancer 1979;44: 340-345 